\title{
EIGENVALUE SUMS OF COMBINATORIAL MAGNETIC LAPLACIANS ON FINITE GRAPHS
}

\author{
JOHN DEVER
}

Abstract. We give a construction of a class of magnetic Laplacian operators on finite directed graphs. We study some general combinatorial and algebraic properties of operators in this class before applying the Harrell-Stubbe Averaged Variational Principle to derive several sharp bounds on sums of eigenvalues of such operators. In particular, among other inequalities, we show that if $G$ is a directed graph on $n$ vertices arising from orienting a connected subgraph of $d$-regular loopless graph on $n$ vertices, then if $\Delta_{\theta}$ is any magnetic Laplacian on $G$, of which the standard combinatorial Laplacian is a special case, and $\lambda_{0} \leqslant \lambda_{1} \leqslant \ldots \leqslant \lambda_{n-1}$ are the eigenvalues of $\Delta_{\theta}$, then for $k \leqslant \frac{n}{2}$, we have

$$
\frac{1}{k} \sum_{j=0}^{k-1} \lambda_{j} \leqslant d-1
$$

Mathematics subject classification (2010): 05C50, 05C20, 05C15, 15B57, 15A42.

Keywords and phrases: Magnetic graph Laplacian, graph Laplacian, eigenvalue inequalities, eigenvalue sums, adjacency matrix, graph spectrum, half-band.

\section{REFERENCES}

[1] E. Beckenbach and R. Bellman, Inequalities, Springer 77, 1965.

[2] E. M. Harrell II AND J. StubBe, On sums of graph eigenvalues, Linear Algebra and its Applications 455, 168-186, 2014.

[3] Y. Higuchi AND T. SHIRAI, A remark on the spectrum of magnetic Laplacian on a graph, Yokohama Math Journal 47, 1999.

[4] E. H. Lieb And M. Loss, Fluxes, Laplacians, and Kasteleyn's theorem, Duke Mathematical Journal 8 (2), 1993, 337-363.

[5] S. Nikolić and G. Kovačević And A. Miličević and N. TRinajstić, The Zagreb indices 30 years after, Croatica Chemica Acta 76 (2), 2003, 113-124.

[6] T. SunADA, Discrete geometric analysis, Geometry on Graphs and its Applications, Proceedings of Symposia in Pure Mathematics, American Mathematical Society 77, 2008, 51-86. 